\title{
Simple and Clear Evidence for Positive Feedback Limitation by Bipolar Behavior during Scanning Electrochemical Microscopy of Unbiased Conductors
}

\author{
Alexander I. Oleinick, ${ }^{\dagger}$ Dario Battistel, ${ }^{\ddagger}$ Salvatore Daniele, ${ }^{\ddagger}$ Irina Svir, ${ }^{\dagger}$ and Christian Amatore, ${ }^{*,+}$ \\ ${ }^{\dagger}$ Département de Chimie, UMR 8640, Ecole Normale Supérieure, 24 Rue Lhomond, F-75231 Paris Cedex 5, France \\ ${ }^{\ddagger}$ Dipartimento di Scienze Molecolari e Nanosistemi, Università Cà Foscari Venezia, Calle Larga S Marta, 213730123 Venice, Italy
}

ABSTRACT: On the basis of an experimentally validated simple theoretical model, it is demonstrated unambiguously that when an unbiased conductor is probed by a scanning electrochemical tip (scanning electrochemical microscopy, SECM), it performs as a bipolar electrode. Though already envisioned in most recent SECM theories, this phenomenon is generally overlooked in SECM experimental investigations. However, as is shown here, this may alter significantly positive feedback measurements when the probed conductor is not much larger than the tip.

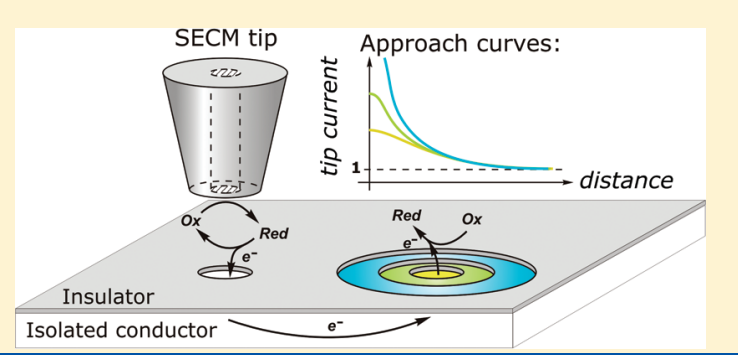

Canning electrochemical microscopy (SECM) has proven to $\checkmark$ be an essential method not only for providing important information on the local topography of scanned samples but also for assessing the local reactivity of substrates at the micrometric scale. ${ }^{1}$ This dual feature has led to the development of SECM applications for a considerable variety of topics ${ }^{1}$ ranging, e.g., from investigations of the topology and electrochemical reactivity of metallic alloys, ${ }^{2 \mathrm{a}, \mathrm{b}}$ organic film patterns, ${ }^{2 \mathrm{c}, \mathrm{d}}$ or deposits for fuel cells $^{2 \mathrm{e}}$ to (bio)chemical catalysis, ${ }^{2 \mathrm{f}-\mathrm{i}}$ photocatalysis, ${ }^{2 \mathrm{j}}$ chemical reactivity, ${ }^{2 \mathrm{k}-\mathrm{m}}$ biological activity of single cells and tissues, ${ }^{2 \mathrm{n}-\mathrm{q}}$ ion transfers at liquid-liquid interfaces, ${ }^{2 \mathrm{r}, \mathrm{s}}$ etc., as well as local microfabrications. ${ }^{2 \mathrm{t}}$

At inert substrates (i.e., not able to consume or produce any electroactive species at their surface), decreasing the SECM tip-substrate distance progressively restricts the access of bulk redox species toward the electroactive tip. ${ }^{1}$ The steady-state tip current, $I_{\mathrm{nf}}$, then decreases vs its value at infinite distance from the substrate (viz., $I_{\text {tip }}^{\infty}=4 n F D C^{\text {bulk }} r_{\text {tip }}$ for a disk tip ${ }^{3} r_{\text {tip }}$ is the tip radius, $n$ the number of electrons per molecule of redox probe exchanged at the tip surface, $C^{\text {bulk }}$ the bulk solution concentration of the probe, and $D$ its diffusion coefficient). This situation, termed "negative feedback", provides exclusively information on the local substrate topology. ${ }^{1}$

Conversely, when the substrate is a suitable conductor, a "positive feedback" occurs due to electrochemical recycling of the redox species by the probed area of the substrate toward the tip. ${ }^{1}$ Experimentally, this positive feedback is then often viewed as being an intrinsic electrochemical property of the substrate area scanned by the tip. However, as has been understood almost since the earliest SECM theories and soundly established in recent ones, ${ }^{4}$ positive feedback features the electrochemical properties of the whole substrate and not only those of the area interrogated by the SECM tip. This surprising feature of a wellfocused "microscopy" is due to the fact that SECM is an active electrochemical method and not a passive one such as the usual microscopies (optical microscopy, scanning tunneling microscopy (STM), atomic force microscopy (AFM), etc.). Hence, to provide a significant recycled flux of the bulk redox species toward the tip, the substrate, as a whole, must be able to efficiently perform as a bipolar electrode. ${ }^{4,5}$ When the substrate is a large nonresistive conductor, this is not a problem and the recycling rate is regulated only by the electrochemical cross-talk between the tip and the substrate area it is probing. ${ }^{4,6,7}$ Since this is the case in most usual circumstances, this characteristic is often extrapolated to conducting substrates of any size, thus leading to a misguided but commonly used view. Indeed, when the substrate is highly resistive (e.g., when composed of poorly connected small metallic grains or elements ${ }^{4 c, e}$ or of a resistive organic film, ${ }^{2 c, d}$ etc.) or has a small area compared to that of the tip, ${ }^{4 a-d, f}$ positive feedback may be significantly limited by the substrate's ability to perform as an efficient bipolar electrode rather than by the electrochemical characteristics of its very probed area. Several theoretical and experimental papers have extensively addressed this issue already, ${ }^{4}$ but in our experience this is still not well perceived by many experimentalists. For this reason we report in this work what we believe is a compelling and very simple theoretical and experimental approach that should unambiguously establish the validity of this important caveat.

\section{EXPERIMENTAL SECTION}

Microfabrication of the Dual-Disk Bipolar Substrates. Figure 1 represents a scanning electron microscopy (SEM) vertical cross-section of the substrate before its localized etching.

Received: March 8, 2011

Accepted: April 30, 2011

Published: May 18, 2011 
It consisted of a layer of platinum $(0.40 \mu \mathrm{m})$ deposited on a silicon wafer by rf magnetron sputtering (MS). A thin insulating layer of alumina $(0.25 \mu \mathrm{m})$ was then deposited by MS on top of the platinum one to insulate electrochemically the platinum conductor from the electrolyte.

The thin alumina layer insulating the platinum substrate surface was then electrochemically etched to define one disk hole of $100 \mu \mathrm{m}$ diameter (nominal dimension). Etching was achieved by hydroxide ions electrochemically generated through the reduction of water by an SECM tip following a previous procedure reported by some of us. ${ }^{8 a}$ The extent of the etched zone was finely controlled by the presence of the weak disodium ethylenediaminetetraacetate acid (noted $\mathrm{H}_{2} \mathrm{Y}^{2-}$ in Figure 2a), which focused the high hydroxide ion concentration below the tip. ${ }^{8}$ This procedure allowed the conducting disks with reproducible dimensions and sufficiently small recesses (vs the disk radii) to be exposed so that any diffusional effects due to them would be negligible. ${ }^{9}$

This procedure was implemented on two different wafers for etching a pair of holes consisting in each case of one hole identical to that in Figure $2 \mathrm{~b}\left(r_{\text {hole }}=50 \pm 5 \mu \mathrm{m}\right.$, designed as the substrate area to be probed by SECM in each series of experiments) coupled with a second hole of a comparable radius $\left(r_{\text {hole }}=25\right.$ or $37.5 \mu \mathrm{m}$, each within a ca. $10 \%$ accuracy) whose ultimate purpose was to serve as the auxiliary cathode of the bipolar substrate. In each case the distance between the pair of exposed platinum disks was ca. $650-700 \mu \mathrm{m}$ to avoid any diffusional interference

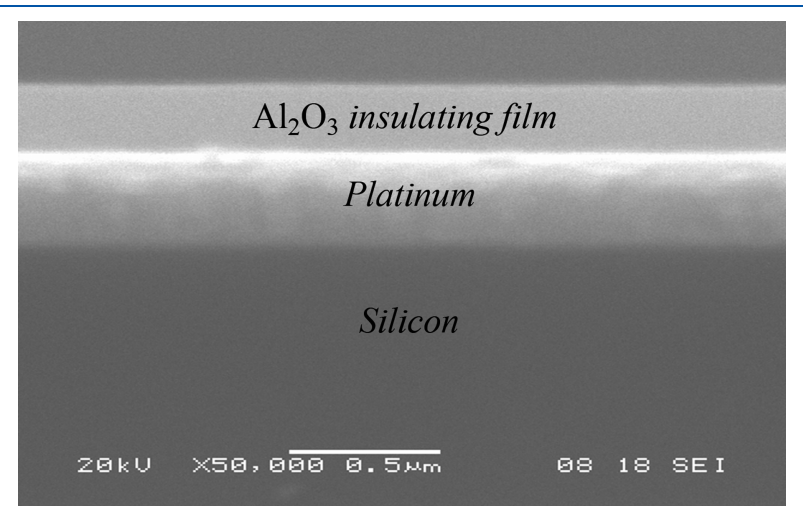

Figure 1. SEM vertical cross-section micrograph of the substrate before its localized etching. between them except those required by the bipolar function, i.e., imposed by the distribution of the bipolar substrate-solution potential difference. ${ }^{4,5}$ Finally, in the third case a single hole was created which was identical to that in Figure $2 \mathrm{~b}$.

SECM Approach Curves on Dual-Disk Bipolar Substrates. A series of approach curves were recorded (i) on the samples before etching (i.e., on the alumina insulator), (ii) after etching a single disk taken as the reference in the following experiments, and (iii) at the reference disk after a second hole was etched to perform as the second pole of the bipolar substrate. The corresponding results are reported in Figure 6.

The probed disk $(50 \pm 5 \mu \mathrm{m}$ radius, Figure $2 \mathrm{~b})$ was made sufficiently large to limit any error due to possible misalignment of its vertical symmetry axis with the SECM tip vertical displacement direction. The remote holes were etched to sizes (diameters of ca. 50 and $75 \mu \mathrm{m}$ ) that were not much larger than that of the SECM tip so that their role in limiting positive feedback currents could be observable at not too short $L$ values (see below) to avoid any significant error in this parameter.

The SECM tip consisted of a Pt microdisk $(12.5 \mu \mathrm{m}$ radius, RG = 10). All approach curves were recorded in $0.1 \mathrm{M} \mathrm{KCl}$ aqueous solutions of $\mathrm{Ru}\left(\mathrm{NH}_{3}\right)_{6}{ }^{3+}, 1 \mathrm{mM}$. The probed substrate was always unbiased, while the tip potential was poised at -0.35 $\mathrm{V}$ vs $\mathrm{Ag} / \mathrm{AgCl}$ to perform on the plateau of the one-electron reduction wave of $\mathrm{Ru}(\mathrm{III})$.

The ensuing approach curves were fitted according to the simple theoretical model developed in this work (see below and the Appendix). It was found that the best fits were obtained for $r_{\text {hole }} / r_{\text {tip }}=2.15$ and 3.20 (rather than 2 and 3, respectively, as expected from the nominal radii in the etching process). These values are within the limits of the $10 \%$ accuracy of the etching procedure (compare Figure $2 b$ ).

\section{RESULTS AND DISCUSSION}

In positive feedback mode, the measured tip current, $I_{\text {tip }}$, is higher than its value, $I_{\text {tip }}^{\infty}$, measured in the same solution bulk. This increase integrates two contrasting effects: a negative one, due to the constrained access of the bulk species to and from the tip as would occur in a "negative feedback" mode for the same configuration, and a positive one stemming from the electrochemical recycling of the redox probe at the substrate surface placed under the SECM tip. Although this is not classical, we may decompose arbitrarily $I_{\text {tip }}$ into two contributions for the sake of
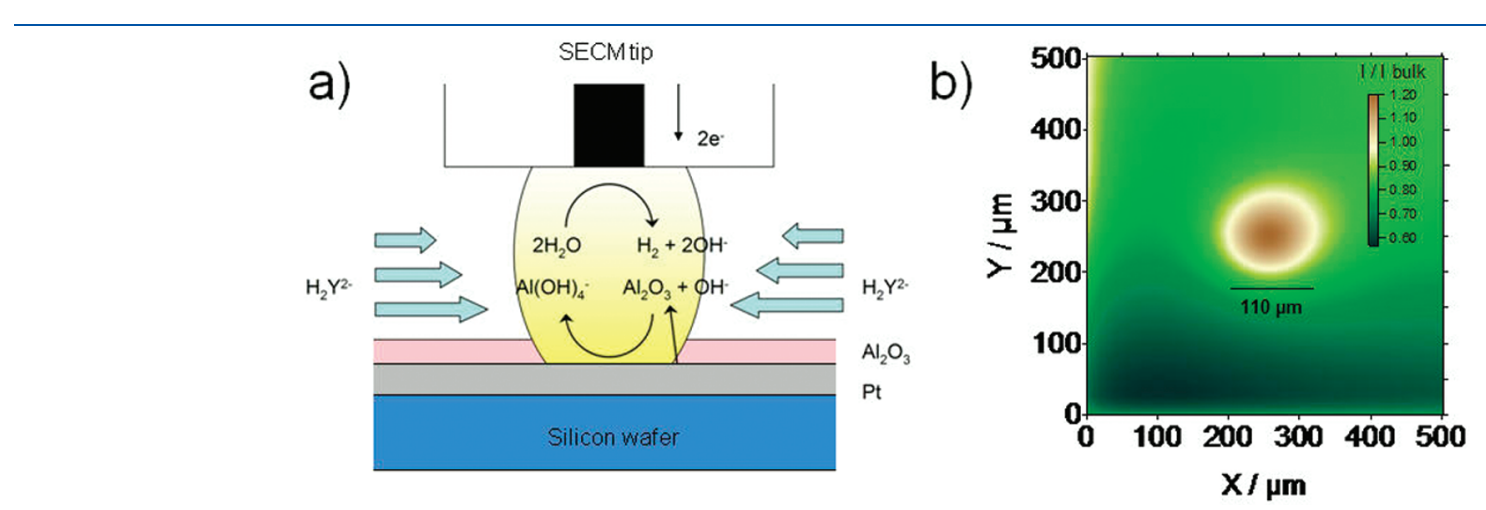

Figure 2. (a) Principle of the localized electrochemical etching of the insulating alumina layer. ${ }^{8 \mathrm{a}}$ (b) SECM scan of the etched platinum disk (100 \pm 10 $\mu \mathrm{m}$ in diameter) exposed in the alumina insulating layer and serving as the probed substrate in this work. SECM scans were recorded in a $1 \mathrm{mM}$ $\mathrm{Ru}\left(\mathrm{NH}_{3}\right)_{6}{ }^{3+}$ in $0.1 \mathrm{M} \mathrm{KCl}$ aqueous solution. The SECM tip radius, $r_{\text {tip }}$, was $12.5 \mu \mathrm{m}$, with $\mathrm{RG}=r_{\text {shaft }} / r_{\text {tip }}=10$, where $r_{\text {shaft }}$ is the radius of the tip shaft (see Figure 4). 

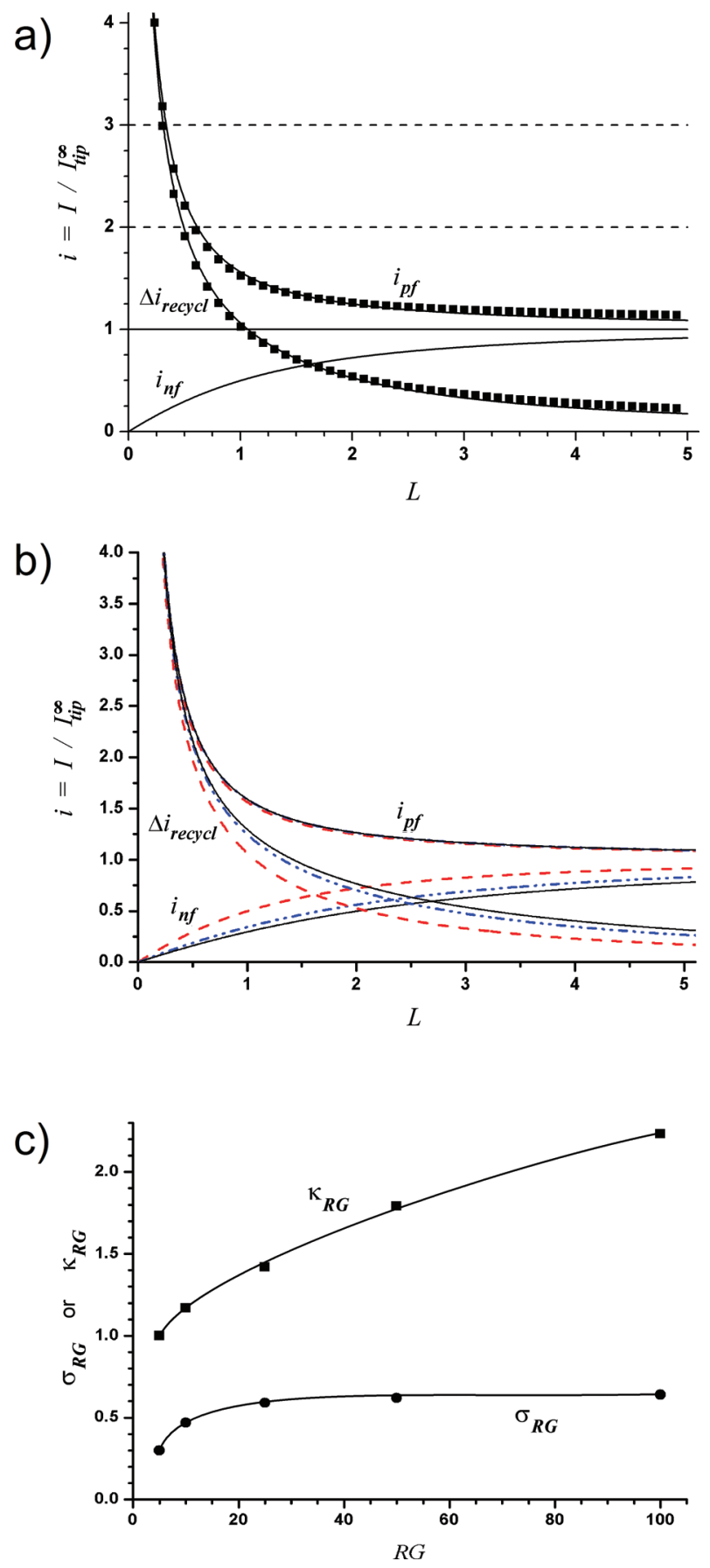

Figure 3. (a) Positive $\left(i_{\text {pf }}\right)$ feedback approach curve plotted on the basis of eqs 3 and 4 (symbols) or published analytical expressions (solid curves) ${ }^{7 \mathrm{~b}, \mathrm{c}}$ for $i_{\mathrm{pf}}$ and $i_{\mathrm{nf}} ; \mathrm{RG}=10$. (b) Variations of $i_{\mathrm{pf}}, \Delta i_{\text {recycl }}$, and $i_{\mathrm{nf}}$ for a series of RG values encompassing the most usual ones: $R G=10$ (dashed), 50 (dashed-dotted), and 100 (solid). (c) Variations of $\sigma_{\mathrm{RG}}$ and $\kappa_{\mathrm{RG}}$ vs RG.

our purpose here:

$$
I_{\text {tip }}=I_{\text {nf }}+\Delta I_{\text {recycl }}
$$

where $\Delta I_{\text {recycl }}$ represents the electrochemical perturbation due to the substrate electroactivity, while $I_{\mathrm{nf}}$ is the contribution which would be observed if the substrate were electrochemically inert (i.e., as in a negative feedback mode) and depends only on the
Table 1. Variations of $\sigma_{\mathrm{RG}}$ and $\kappa_{\mathrm{RG}}$ vs RG

\begin{tabular}{cccccc} 
& $\mathrm{RG}=5$ & $\mathrm{RG}=10$ & $\mathrm{RG}=25$ & $\mathrm{RG}=50$ & $\mathrm{RG}=100$ \\
$\sigma_{\mathrm{RG}}$ & 0.31 & 0.47 & 0.59 & 0.62 & 0.64 \\
$\kappa_{\mathrm{RG}}$ & 1.00 & 1.17 & 1.42 & 1.78 & 2.23 \\
\hline
\end{tabular}

topology of the tip-substrate assembly. Whenever the substrate may perform as a perfect collector, ${ }^{6}$ at tip-substrate distances, $l$, small enough for $I_{\text {nf }}$ to be negligible, ${ }^{1,4,7} I_{\text {tip }}$ is expected to tend toward the current value observed in the thin-layer cell composed by the two facing electrodes. If the substrate is much wider than the tip, this limiting value should be controlled only by the tip surface area and the tip-substrate distance. Hence ${ }^{10}$

$$
\Delta I_{\text {recycl }} \rightarrow\left(\Delta I_{\text {recycl }}\right)^{\text {lim }}=n F D C^{\text {bulk }}\left(\pi r_{\text {tip }}^{2}\right) / l
$$

In the following, we comply with the classical practice of normalizing current values relative to $I_{\text {tip }}^{\infty}$ and any distance values relative to $r_{\text {tip. }}$. Hence, eq 1 is rewritten as

$$
i_{\text {tip }}=i_{\text {nf }}+\Delta i_{\text {recycl }}
$$

Numerical simulations and comparison with reported empirical analytical solutions ${ }^{7 \mathrm{~b}, \mathrm{c}}$ for $i_{\mathrm{pf}}$ and $i_{\mathrm{nf}}$ showed (see the Appendix) that for a large perfect collecting substrate probed by a tip equipped with a sufficiently thick insulating shaft $\left(r_{\text {shaft }}=r_{\text {tip }}(\mathrm{RG})\right), \Delta i_{\text {recycl }}$ obeys the following empirical analytical formulation over the range of $L=l / r_{\text {tip }}$ values of experimental interest:

$$
\Delta i_{\text {recycl }}=\frac{\pi}{4 L}+\sigma_{\mathrm{RG}}\left[1-\exp \left(-\kappa_{\mathrm{RG}} \frac{\pi}{4 L}\right)\right] \underset{L \rightarrow 0}{\longrightarrow}\left(\Delta i_{\text {recycl }}\right)^{\lim }=\frac{\pi}{4 L}
$$

where $\sigma_{\mathrm{RG}}$ and $\kappa_{\mathrm{RG}}$ are two constants whose values depend only on $\mathrm{RG}$ (e.g., $\sigma_{\mathrm{RG}}=0.62$ and $\kappa_{\mathrm{RG}}=1.78$ for $\mathrm{RG}=50$ or $\sigma_{\mathrm{RG}}=0.47$ and $\kappa_{\mathrm{RG}}=1.17$ for $\mathrm{RG}=10$ ). Figure 3 illustrates the excellent accuracy of eqs 3 and 4 as well as the variations of $\sigma_{\mathrm{RG}}$ and $\kappa_{\mathrm{RG}}$ with RG (Table 1).

The main interest of the formulation in eqs 3 and 4 is that it enables evaluation in a compelling way of the consequence of the substrate bipolar behavior on the positive feedback magnitude. Indeed, the existence of a recycling contribution, $\Delta i_{\text {recycl }}$ monitored by the SECM tip implies that a significant current, $-\Delta i_{\text {recycl }}$ may flow from the substrate placed under the tip. However, an unbiased conductor cannot deliver any net current, so the substrate must perform as a bipolar electrode submitted to a concentration gradient $t^{4,5}$ and consequently must deliver a current $+\Delta i_{\text {recycl }}$ resulting from its electroactivity outside the range covered by the tip. This has already been recognized in several theoretical papers and even investigated experimentally in a few instances. ${ }^{4}$

Whenever this bipolar operation is not feasible or not possible to the required extent, the $\Delta i_{\text {recycl }}$ (hence $i_{\text {tip }}$ ) value will be limited not necessarily by the electrochemical properties of the scanned substrate area but by its inability to perform as a bipolar electrode as efficiently as required. ${ }^{4 \mathrm{~d}-\mathrm{f}}$ This is necessarily true even in the absence of sluggish charge transfer or other electrochemical factors limiting $i_{\text {tip }}$ in the positive feedback mode. ${ }^{4 f, 11}$

To characterize unambiguously this effect on positive feedback approach curves, we resorted to a specifically designed system in which the probed substrate consisted of one platinum disk microelectrode possibly connected electrically to another remote one of variable radius, $r_{\text {hole }}$, and placed far apart (see Figure 4). The probed disk radius was kept constant (ca. $50 \mu \mathrm{m}$ ), 


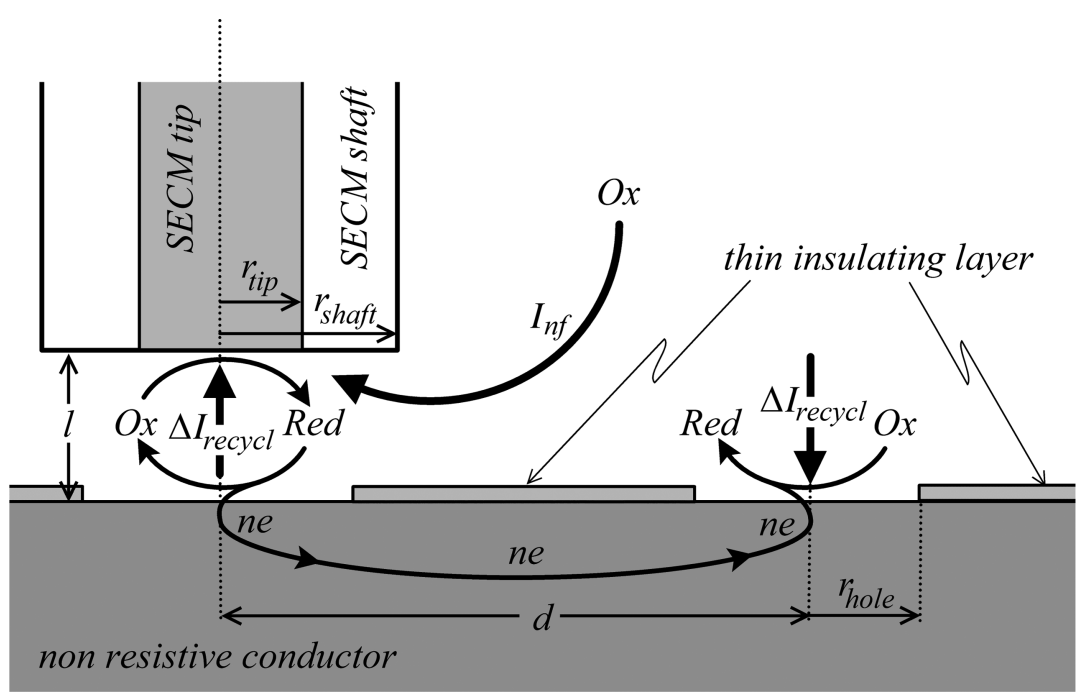

Figure 4. Sketch of a cross section of the system under scrutiny along the plane passing through the vertical axes of the two disks etched in the insulating cover of the substrate with indication of a positive feedback mechanism.

not being excessively larger than that of the tip $(12.5 \mu \mathrm{m})$ to represent a small-sized substrate situation. The distance $d$ $(650-700 \mu \mathrm{m})$ between the disk pair was fixed so that $d \gg$ $r_{\text {shaft }}$ and $d \gg r_{\text {hole }}$ in order that any concentration gradient crosstalk between the disk pair was negligible beyond that imposed by the bipolar behavior (Figure 4). Experimentally, such dual-disk substrates were readily constructed by electrochemical etching of two disk holes through a nanometric insulating film of alumina deposited over a comparatively thick flat platinum substrate ${ }^{8 a}$ (see the Experimental Section).

Let us assume that the bulk solution initially contains only one redox species, Ox, prone to be reduced into Red at the SECM tip. At the steady state, the scanned disk of the substrate then probes a solution enriched with Red and may then perform as an anode (viz., oxidizing Red back into Ox)..$^{1,4,5,7,11}$ However, the corresponding oxidation current, $-\Delta i_{\text {recycb }}$ needs to be compensated by a cathodic one (viz., $+\Delta i_{\text {recycl }}$ ) which can be delivered only by means of $\mathrm{Ox}$ reduction. ${ }^{4,5}$ When the tip-substrate distance $L$ is sufficiently small for no significant $\mathrm{Ox}$ concentration to be present at the surface of the scanned disk, ${ }^{7}$ the cathodic pole may only consist of the remote substrate disk which is exposed to the Ox bulk solution. Hence, $\Delta i_{\text {recycl }}$ cannot exceed the value of the limiting diffusional current which may flow at this remote disk, so that

$$
\Delta i_{\text {recycl }} \leq\left|i_{\text {hole }}^{\max }\right|=4 n F D C^{\text {bulk }} r_{\text {hole }} / I_{\text {tip }}^{\infty}=r_{\text {hole }} / r_{\text {tip }}
$$

Under most experimental conditions, when $L \ll 1$ and the substrate performs as a perfect collector, $i_{\text {tip }}$ is expected to reach values of several units. ${ }^{1,4,7}$ However, because of the inequality in eq 5 , in the present system, the fulfillment of this fact implies that $r_{\text {hole }} / r_{\text {tip }}$ is large enough compared to $\left(\Delta i_{\text {recycl }}\right)^{\lim }=(\pi / 4) r_{\text {tip }} / l$ as follows from eq 2 . However, since $r_{\text {hole }} / r_{\text {tip }}$ is fixed by the system construction while $\left(\Delta i_{\text {recycl }}\right)^{\lim }$ increases drastically when $l \rightarrow 0$, the inequality in eq 5 dictates that, as soon as $L<L^{\min }=(\pi / 4) \times$ $r_{\text {tip }} / r_{\text {hole }} \Delta i_{\text {recycl }}$ is necessarily limited by the performance of the remote disk rather than by the electrochemical properties of the probed one. Figure 5a illustrates this contingency for the two values of $r_{\text {hole }} / r_{\text {tip }}$ corresponding to the experimental cases investigated here for the $\Delta i_{\text {recycl }}$ component.

Noteworthy, the corresponding total positive feedback currents in Figure 5 b display a smooth maximum at small $L$, a feature that is often observed but discarded, being ascribed to a lack of precision on $L$ and to tilt effects. ${ }^{12}$ The present analysis shows that such a maximum is a normal feature as soon as the surface area of the scanned conductor is not large enough in comparison to that of the tip. This maximum results from the superimposition of a leveling component, $\Delta i_{\text {recycl }}$ smoothly reaching its horizontal limit (compare Figure 5a) and of a negative feedback component, $i_{n f}$, which tends toward zero with its largest slope when $L \rightarrow 0$ (compare Figure 3).

To demonstrate in a clear and unambiguous way the experimental validity of this limitation, a series of positive feedback approach curves were recorded and compared to the above theoretical predictions for different values of $r_{\text {hole }} / r_{\text {tip }}$ at values close to unity (i.e., 2 and 3; see the symbols in Figure 6). Note that the range of $r_{\text {hole }} / r_{\text {tip }}$ values was purposely imposed to be not much larger than unity so that the corresponding limits, $L^{\mathrm{min}}=l^{\mathrm{min}} / r_{\text {tip }}$, could be observable experimentally over a range of $L$ values where the accuracy on the tip-substrate distance was sufficiently good. ${ }^{12}$ As evidenced, the agreement between experiments and predictions (see the Appendix) is excellent.

For $r_{\text {hole }} / r_{\text {tip }}=0$ (i.e., when no remote disk was present), the simple model developed here could not be used quantitatively, but it should be noted that similar shapes have been predicted previously for SECM at an unbiased substrate of small size. ${ }^{4 \mathrm{~d}}$ However, the present model still allows rationalization of the shape of the corresponding approach curve in Figure 6. The almost perfect negative feedback approach curve monitored before any hole was etched onto the substrate (open circles in Figure 6) is evidence that the peculiar shape of the approach curve monitored for $r_{\text {hole }} / r_{\text {tip }}=0$ cannot be ascribed to any significant leaks through the alumina layer. This approach curve thus stems only from the diffusional behavior created by the tip action over the probed single disk hole. Under such conditions, the substrate cannot behave as an efficient bipolar electrode because its edge (i.e., the cathodic pole) is exposed to a depleted concentration of Ox. Hence, $\Delta i_{\text {recycl }}$ is much smaller than it would be for a substrate of infinite size though it nevertheless has a non-negligible value. It is noteworthy that $\Delta i_{\text {recycl }}$ experiences a maximum (at ca. $L=0.6$ ) and tends toward 0 when $L \rightarrow 0$ and $L \rightarrow \infty$. Indeed, when $L \rightarrow 0$, the solution near the probed disk surface 
a)

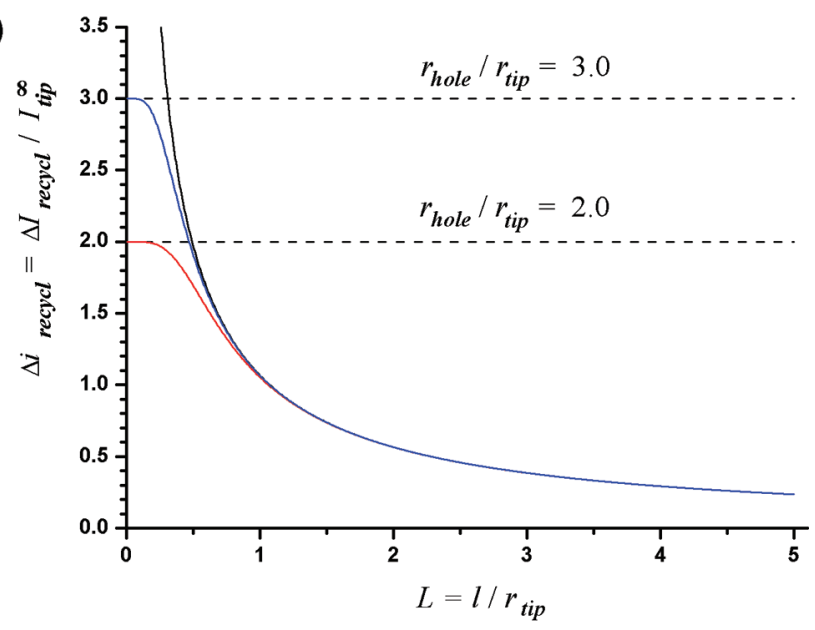

b)

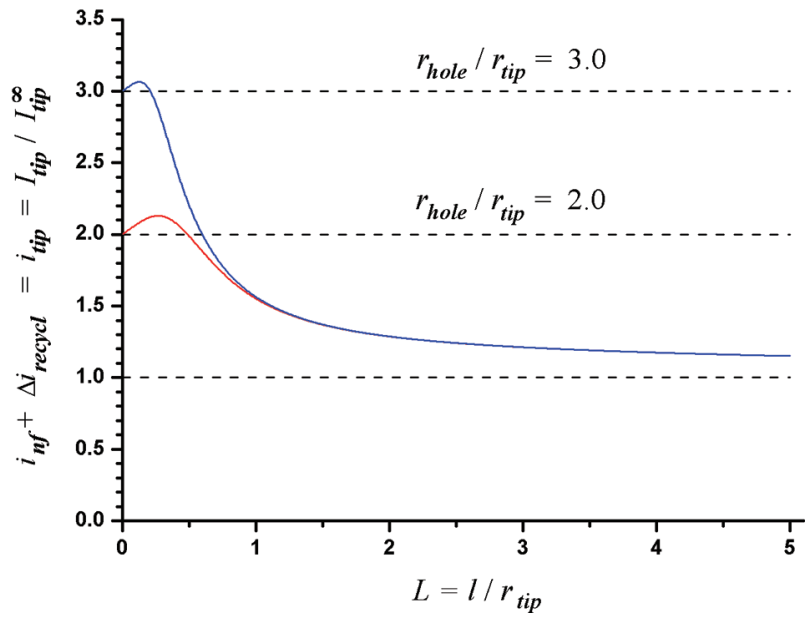

Figure 5. (a) Predicted variations of $\Delta i_{\text {recycl }}$ vs $L$ according to the $[4 / 5]$ two-point Pade approximation based on the limits discussed in the text (see the Appendix) $(\mathrm{RG}=10)$. From bottom to top, $r_{\text {hole }} / r_{\text {tip }}=2.0$ (red), 3.0 (blue), and $\infty$ (black). Dashed horizontal lines represent the limits at $r_{\text {hole }} / r_{\text {tip }}$ in each case. (b) Predicted positive feedback $i_{\text {tip }}=i_{\text {nf }}+$ $\Delta i_{\text {recycl }}$ approach curves for the system under scrutiny, where $\Delta i_{\text {recycl }}$ values are taken from (a) and $i_{\mathrm{nf}}$ is from ref $7 \mathrm{c}$.

cannot be replenished in $\mathrm{Ox}$ as is evidenced by the fact that the negative feedback contribution decays to zero (see Figure 3). Then, no reduction current may flow because the would-be cathodic pole of the substrate is exposed only to Red over its whole surface area, i.e., $\Delta i_{\text {recycl }} \rightarrow 0$. On the other hand, no significant positive feedback also occurs at high $L$ values because the tip-probed disk configuration is then too loose to allow any significant collection by the substrate. ${ }^{13}$ Conversely, at intermediate $L$ values the collection efficiency is small but still significant so that the central part of the probed disk substrate may be exposed to a solution enriched in Red (i.e., it may perform as an anode) while its edge remains in contact with a suitable concentration of $\mathrm{Ox}$ (i.e., it may perform as a cathode). Hence, at intermediate $L$ values a small but significant bipolar operation is feasible so that $\Delta i_{\text {recycl }}$ experiences a maximum in this range.

\section{CONCLUSION}

The simple theoretical and experimental model developed in this work is unambiguous evidence that when an isolated

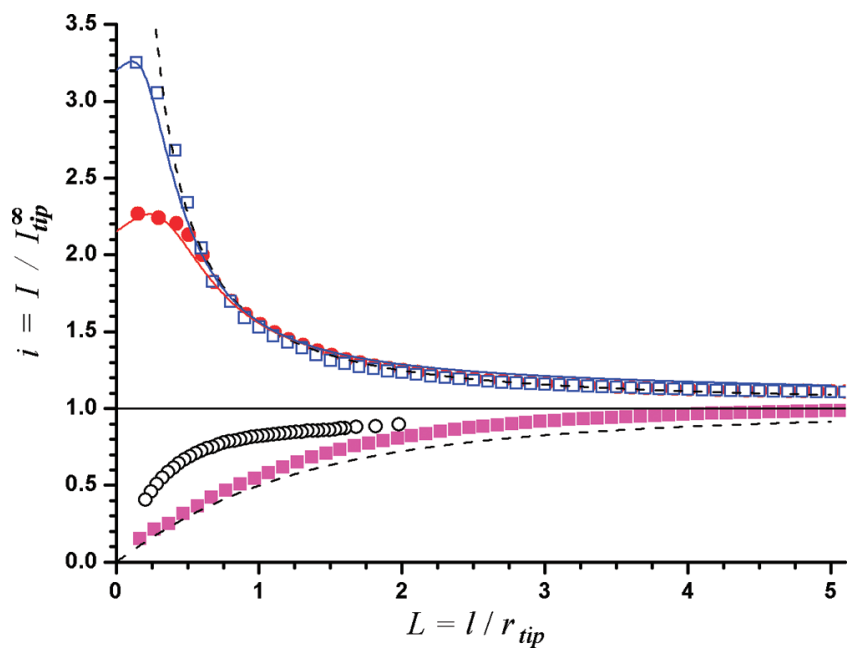

Figure 6. Comparison between experimental (symbols) and theoretical (full lines) approach curves (tip diameter $25 \mu \mathrm{m}, \mathrm{RG}=10$ ). Substrate: nominal diameter of the scanned platinum disk, $100 \pm 10 \mu \mathrm{m}$; auxiliary platinum disk diameters $(\mu \mathrm{m})$ : 0 (open black circles; i.e., no auxiliary disk), $50 \pm 5$ (filled red circles), and $75 \pm 8$ (open blue squares). Note that the best fits correspond to $r_{\text {hole }} / r_{\text {tip }}=2.15$ (filled circles and red curve) and 3.20 (open squares and blue curve), which match the nominal radii within their accuracy (compare Figure $2 b$ ). Dashed lines feature negative and positive feedback currents when the bipolar limitation is neglected. A negative feedback approach curve (filled squares) was also recorded over the intact insulating alumina film. $\mathrm{Ru}\left(\mathrm{NH}_{3}\right)_{6}{ }^{3+}, 1 \mathrm{mM}$, in $0.1 \mathrm{M} \mathrm{KCl}$. The tip potential was poised on the plateau of the $\mathrm{Ru}(\mathrm{III})$ reduction wave $(-0.35 \mathrm{~V}$ vs $\mathrm{Ag} / \mathrm{AgCl})$.

conductor is probed by a scanning electrochemical tip, it needs to perform as a bipolar electrode to allow any significant positive feedback. This phenomenon may significantly alter electrochemical measurements when the probed conductor is not much larger than the tip because its bipolar performance is then limited due to the concentration distribution in the tip-substrate cleft. In this work we voluntarily restricted the theoretical and experimental illustration of such a limitation to the case of approach curves along the axis of symmetry of the probed substrate disk, yet the effect which was thus unambiguously established is evidently also sensible when lateral scans are performed. See, for example, detailed effects of this limitation on lateral scans in published works by Amemiya ${ }^{4 e}$ or by Zoski. $^{4 c}$

This limits in turn the recycling efficiency due to the scanned surface area of the conductor. This caveat is thus of extreme importance when using SECM to test the conductive or electrochemical properties of substrates of small sizes. ${ }^{4}$

Conversely, when a highly conducting substrate extends well beyond the area covered by the tip, the constraints due to the bipolar function become negligible because the large external parts of the substrate may act as an efficient second pole of the bipolar substrate. Indeed, these large external parts of the substrate may perform at very small current densities while compensating the high current density due to the redox recycling occurring at the scanned section of the substrate. This explains why SECM theories which neglect the bipolar mode constraints have nonetheless proven to be extremely useful for the treatment of experimental data monitored at substrates with large sizes compared to the tip size. ${ }^{1,7}$ 
APPENDIX: PREDICTION OF THE APPROACH CURVES USED IN THIS WORK

Validation of the Formulation in eqs 3 and 4. The formulation in eqs 1 and 3 is unusual compared to the existing theories of SECM in the fact that it posits that positive feedback currents have two main contributions. One reflects the hindrance to the diffusional communication between the tip and the bulk solution, being caused by the proximity of the SECM tip to the substrate surface; the second represents the result, $\Delta i_{\text {recycl }}$ of the diffusional cross-talk established between the tip and the redoxactive substrate.

In first approximation, the former contribution is reflected by the negative feedback current, $i_{\text {nf }}$, which would be observed for a nonconducting substrate. Evidently, identifying this contribution with $i_{\text {nf }}$ when a positive feedback is occurring is not strictly correct because this amounts to the assumption that, at the steady state, concentration profiles obey the principle of superposition, which is false because the specific electrochemical boundary conditions cannot be separated as independent functions. However, at sufficiently small tip-substrate distances the intertwining terms become extremely small, so each contribution becomes nearly independent of the other. Since the main experimental interest of SECM involves situations when the tip-substrate distances are small enough to allow significant feedback, this approximate formulation may be considered as formally valid. Furthermore, any small residual bias introduced by it is transferred into the term $\Delta i_{\text {recycl }}$.

Because of the above, the limit of $\Delta i_{\text {recycl }}$ at small $L$ values is certainly given as in eq 4 since then the diffusion communication with the solution is fully suppressed as evidenced by the fact that $i_{\text {nf }}$ tends to zero when $L$ does so as well, ${ }^{4 \mathrm{a}, \mathrm{b}, \mathrm{d}, 7}$ yet $\Delta i_{\text {recycl }}$ necessarily deviates from this limit at larger $L$ values. To evaluate these deviations, we resorted to previously published ${ }^{7 \mathrm{~b}, \mathrm{c}}$ empirical analytical formulations for $i_{\text {nf }}$ and $i_{\mathrm{pf}}$ at a perfect inert or collector substrate (respectively) of infinite size and evaluated $\Delta i_{\text {recycl }}$ as their difference, viz., $\Delta i_{\text {recycl }}=i_{\text {pf }}-i_{\text {nf }}$ (as shown in Figure $3 \mathrm{a}$ for $\mathrm{RG}=10$ ). Figure $3 \mathrm{~b}$ represents the result of this approach for other RG values.

It is interesting to note that, in a positive feedback mode when the substrate has an infinite size, $i_{\text {tip }}$ does not depend significantly on RG (Figure $3 \mathrm{~b}){ }^{7}$ while $\Delta i_{\text {recycl }}$ does depend severely on this parameter as does $i_{\text {nf }}$ also. ${ }^{7}$ In fact, this feature provides further physical support to the formulation in eqs 1 and 3 . Indeed, $i_{\mathrm{nf}}$ varies significantly with RG because the larger the insulating shaft radius, the more severely the diffusion from/to the bulk to/from the tip is hampered. The same diffusional hindering increases the collection efficiency in the tip-substrate assembly, viz., $\Delta i_{\text {recycl }}$ because Red produced by the tip also becomes highly confined into the cleft between the tip and the substrate. Hence, it is normal to observe that the effects of RG on $i_{\mathrm{nf}}$ and $\Delta i_{\text {recycl }}$ are nearly opposite, so they almost compensate each other and $i_{\text {tip }}$ in a positive feedback mode is almost independent of RG. This particular fact illustrates the great benefit of the present formulation of $i_{\text {tip }}$ beyond its specific use in this work.

In eq 4, the bracketed expression represents the coupling term due to the partial cross-talk between the phenomena occurring in the bulk solution and the redox recycling between the tip and the substrate. This term becomes rapidly negligible as soon as $L \ll$ $\pi \kappa_{\mathrm{RG}} / 4$, e.g., as soon as $L \ll 1.4$ for $\mathrm{RG}=50$, i.e., within the range where eq 4 is useful for our purposes here. Hence, one can consider that, though not rigorously valid in the mathematical sense, the formulation in eqs 3 and 4 is valid over the whole range of experimental interest for most investigations using SECM positive feedback currents.

Evaluation of $\Delta i_{\text {recycl }}$ for the Bipolar Dual-Disk Systems Investigated in This Work. The discussion detailed in the main text describes the origin of the constraint which may limit $\Delta i_{\text {recycl }}$ when the ability of the scanned substrate to perform in an efficient bipolar mode is inadequate. For the dual-disk systems investigated here, when $L<L_{\min }=(\pi / 4) r_{\text {tip }} / r_{\text {hole }}$, one obtains $i_{\text {tip }}$ $=\left(r_{\text {hole }} / r_{\text {tip }}\right)+i_{\text {nf }}$, while $i_{\text {tip }}$ remains given by eqs 3 and 4 for $L \gg$ $L_{\text {min }}$ as occurs for a perfect collector substrate. ${ }^{7}$ The simplicity of the present model does not allow rigorous prediction of the variations of $\Delta i_{\text {recycl }}$ with $L$ around $L_{\text {min }}$. We thus resorted to a two-point Pade approximation ${ }^{14}$ which consists in approximating any function $f(L)$ whose limits are known on both sides of a given value (i.e., $L_{\min }$ in our case) by the following function $f_{[m / n]}(L)$ :

$$
f_{[m / n]}(L)=\frac{p_{0}+p_{1} L+\ldots+p_{m}{ }^{L m}}{1+q_{0} L+\ldots+q_{n} L^{n}}
$$

where the coefficients $p_{i}$ and $q_{j}$ are derived through the requirement that $f_{[m / n]}(L)$ converges toward its limits at $L \ll L_{\min }$ and $L \gg L_{\min }$; the subscript $[\mathrm{m} / \mathrm{n}]$ indicates the level of approximation of the Pade function $f_{[m / n]}(L)$. In the present case an $[m=4 /$ $n=5]$ level of approximation was found sufficient for all our purposes. Figure 6 shows the ensuing variations of $\Delta i_{\text {recycl }}$ with $L$ for $r_{\text {hole }} / r_{\text {tip }}=2.15,3.20$, and $\infty$ for $R G=10$. Note that very similar curves would be obtained for other common values of RG.

\section{AUTHOR INFORMATION}

\section{Corresponding Author}

*E-mail: christian.amatore@ens.fr.

\section{ACKNOWLEDGMENT}

In Paris, this work was supported in part by the CNRS (UMR 8640 "PASTEUR"), Ecole Normale Supérieure (ENS), University Pierre and Marie Curie (UPMC), and the French Ministry of Research. In Venice, this work was supported by the Italian Ministry of Research (MUR, PRIN 2008 MWHCP2). I.S. thanks ANR (Chaire d'Excellence "MicroNanoChem") and CNRS for a Directeur de Recherche position in ENS (UMR 8640).

\section{REFERENCES}

(1) For reviews on SECM see, e.g.: (a) Bard, A. J.; Fan, F. R; Mirkin, M. V. In Physical Electrochemistry: Principles, Methods and Applications; Rubinstein, I., Ed.; Marcel Dekker: New York, 1995; Chapter 5, pp 209-242. (b) Bard, A. J., Mirkin, M. V., Eds. Scanning Electrochemical Microscopy; Marcel Dekker: New York, 2001. (c) Pust, S.; Maier, W.; Wittstock, G. Z. Phys. Chem. (Muenchen) 2008, 222, 1463-1517.

(2) Owing to the large number of significant studies using SECM, it is impossible to cite them all, yet the following exemplify the wide variety of experimental topics and main groups concerned: (a) Salles, M. O.; Battistel, D.; Lima, A. S.; Bertotti, M.; Daniele, S. Electroanalysis 2011, 23, 595-603. (b) McKelvey, K.; Edwards, M. A.; Unwin, P. R. Anal. Chem. 2010, 82, 6334-6337. (c) Leroux, Y.; Schaming, D.; Ruhlmann, L.; Hapiot, P. Langmuir 2010, 26, 14983-14989. (d) Zhang, J.; Burt, D. P.; Whiworth, A. L.; Mandler, D.; Unwin, P. Phys. Chem. Chem. Phys. 2009, 11, 3490-3496. (e) Eckhard, K.; Chen, X.; Turcu, F.; Schuhmann, W. Phys. Chem. Chem. Phys. 2006, 8, 5359-5365. (f) Rodriguez-Lopez, 
J.; Minguzzi, A.; Bard, A. J. J. Phys. Chem. C 2010, 114, 18645-18655. (g) Oliveira, E. M.; Beyer, S.; Heinze, J. Bioelectrochemistry 2007, 71, 186-191. (h) Turcu, T.; Schulte, A.; Hartwich, G.; Schuhmann, W. Biosens. Bioelectron. 2004, 20, 925-932. (i) Takahashi, Y.; Shevchuk, A. I.; Novak, P.; Murakami, Y.; Shiku, H.; Korchev, Y. E.; Matsue, T. J. Am. Chem. Soc. 2010, 132, 10118-10126. (j) Ye, H.; Lee, J.; Jang, J. S.; Bard, A. J. J. Phys. Chem. C 2010, 114, 13322-13328. (k) Ding, Z.; Quinn, B. M.; Bard, A. J. J. Phys. Chem. B 2001, 105, 6367-6374. (1) Zigah, D.; Noel, J. M.; Lagrost, C.; Hapiot, P. J. Phys. Chem. C 2010, 114, 3075-3081. (m) Georganopoulou, D. G.; Mirkin, M. V.; Murray, R. W. Nano Lett. 2004, 4, 1763-1767. (n) Schulte, A.; Nebel, M.; Schuhmann, W. Annu. Rev. Anal. Chem. 2010, 3, 299-318. (o) Schulte, A.; Schuhmann, W. Angew. Chem., Int. Ed. 2007, 46, 8760-8777. (p) Sun, P.; Laforge, F. O.; Abeyweera, T. P.; Rotenberg, S. A.; Carpino, J.; Mirkin, M. V. Proc. Natl. Acad. Sci. U.S.A. 2008, 105, 443-448. (q) Amatore, C; Arbault, S.; Guille, M.; Lemaitre., F. Chem. Rev. 2008, 108, 2585-2621. (r) Shao, Y.; Mirkin, M. V. J. Phys. Chem. B 1998, 102, 9915-9921. (s) Hatay, I.; Bin, S.; Mendez, M. A.; Corminboeuf, C.; Khoury, T.; Gros, C. P.; Bourdillon, M.; Meyer, M.; Barbe, J. M.; Ersoz, M.; Zalis, S.; Samec, Z.; Girault, H. H. J. Am. Chem. Soc. 2010, 132, 13733-13741. (t) Szunerits, S.; Knorr, N.; Calemczuk, R.; Livache, T. Langmuir 2004, 20, 9236-9241.

(3) (a) Saito, Y. Rev. Polarogr. Jpn. 1968, 15, 177-187.(b) Amatore, C. In Physical Electrochemistry: Principles, Methods and Applications; Rubinstein, I., Ed.; Marcel Dekker: New York, 1995; Chapter 4, pp 131-208.

(4) (a) Wipf, D. O.; Bard, A. J. J. Electrochem. Soc. 1991, 138, 469-474. (b) Bard, A. J.; Mirkin, M. V.; Unwin, P. R.; Wipf, D. O. J. Phys. Chem. 1992, 96, 1861-1868. (c) Zoski, C. G.; Simjee, N.; Guenat, O.; Kudelka-Hep, M. Anal. Chem. 2004, 76, 62-72. (d) Xiong, H.; Guo, J.; Amemiya, S. Anal. Chem. 2007, 79, 2735-2744. (e) Kim, E.; Kim, J.; Amemiya, S. Anal. Chem. 2009, 81, 4788-4791. (f) Nioradze, N.; Kim, J.; Amemiya, S. Anal. Chem. 2011, 83, 828-835.

(5) (a) Amatore, C.; Brown, A. R.; Thouin, L.; Warkocz, J. S. C. R. Acad. Sci., Ser. IIc 1998, 1, 509-515. (b) Amatore, C.; Thouin, L.; Warkocz, J. S. Chem.-Eur. J. 1999, 5, 456-465. (c) For a similar situation enforced within a confined microfluidic channel compare the following: Fosdick, S. E.; Crooks, J. A.; Chang, B. Y.; Crooks, R. M. J. Am. Chem. Soc. 2010, 132, 9226-9227.

(6) We use the term "perfect collector" to enforce the view that it may perform over its whole probed area of importance for the diffusional coupling with the tip as a biased collecting electrode whose potential is poised on the redox plateau of the species to be recycled ("Red" in Figure 4$).^{4 \mathcal{C}}$ This corresponds to the boundary condition imposed in the present theories of SECM developed for reversible charge transfers and infinitely sized conducting substrates.

(7) See, e.g.: (a) Sklyar, O.; Kueng, A.; Kranz, C.; Mizaikoff, B.; Lugstein, A.; Bertagnolli, E.; Wittstock, G. Anal. Chem. 2005, 77, 764-771. (b) Lefrou, C. J. Electroanal. Chem. 2006, 592, 103-112. (c) Cornut, R.; Lefrou, C. J. Electroanal. Chem. 2007, 608, 59-66.

(8) (a) Battistel, D.; Daniele, S.; Gerbasi, R.; Baldo, M. A. Thin Solid Films 2010, 518, 3625-3631. For previous reports of a similar concentration-focusing strategy used in another context, see:(b) Tian, Z. W.; Feng, Z. D.; Tian, Z. Q.; Zhuo, X. D.; Mu, J. Q.; Li, C. Z.; Lin, H. S.; Ren, B.; Xie, Z. X.; Hu, W. L. Faraday Discuss. 1992, 94, 37-44. (c) Zhang, L.; Ma, X. Z.; Zhuang, J. L.; Qiu, C. K.; Du, C. L.; Tang, J.; Tian, Z. W. Adv. Mater. 2007, 19, 3912-3918.

(9) Amatore, C.; Oleinick, A. I.; Svir, I. Anal. Chem. 2009, 81, 4397-4405.

(10) Bard, A. J.; Faulkner, L. R. Electrochemical Methods, John Wiley \& Sons: New York, 2001; p 454.

(11) Cornut, R.; Lefrou, C. J. Electroanal. Chem. 2008, 621, $178-184$.

(12) For a description of the experimental difficulty of precisely knowing the tip-substrate distance at short $L$ values and avoiding tilt angles, see, e.g.: Nebel, M.; Eckhard, K.; Erichsen, T.; Schulte, A.; Schuhmann, W. Anal. Chem. 2010, 82, 7842-7848.

(13) (a) Schroeder, T. J.; Jankowski, J. A.; Kawagoe, K. T.; Wightman, R. M.; Lefrou, C.; Amatore, C. Anal. Chem. 1992, 64, 3077-3083. (b) Amatore, C.; Szunerits, S.; Thouin, L.; Warkocz, J. S. J. Electroanal. Chem. 2001, 500, 62-70.

(14) Baker, G.; Graves-Morris, P. Pade Approximants (Encyclopedia of Mathematics and Its Applications), 2nd ed.; Cambridge University Press: Cambridge, U.K., 1996; p 764. 